\title{
Félix Plater y la enfermedad de Dupuytren
}

\author{
J. M. ${ }^{\text {a }}$ Arandes Renú ${ }^{(1)}$, A. Bové Boada ${ }^{(2)}$ \\ ${ }^{(I)}$ SeRVICIO DE Cirugía ORTOPÉdica Y TRAUMatología. \\ ${ }^{(2)}$ SerVicio de MEdicina InTERNA. Hospital CLÍNIC. BarCELONA.
}

\author{
Correspondencia: \\ Dr. José $\mathrm{M}^{\mathrm{a}}$ Arandes Renú \\ C/. Dr. Roux, 12, 4을 $2^{\mathrm{a}}$ \\ 08017 Barcelona \\ Tfno. 932044240 \\ Fax: 932044240 \\ e-mail: 6521jar@comb.es
}

\begin{abstract}
Es difícil identificar la primera descripción en la literatura médica de la enfermedad de Dupuytren, posiblemente sea la de Félix Plater en el año 1614, pero el texto está escrito en un latín lleno de modismos y no queda claro si considera que la causa de la retracción son los tendones flexores u otra estructura cordonal formada en la palma de la mano.
\end{abstract}

Palabras clave: Enfermedad de Dupuytren, historia, Félix Plater.
Félix Plater and the Dupuytren's Disease. It is difficult to identify the first description in the medical literature of the Dupuytren's Disease, maybe it was those from Felix Plater in 1614, but the text is written in such a full of Latin idioms it is not clear if he considers the retraction caused by flexor tendons or by any other chordal structure in hands palm.

Key words: Dupuytren's Disease, history, Félix Plater

Rev. Iberam. Cir. Mano - Vol. 30 - Núm. 64 - Abril 2004 (23-24)

T a enfermedad de Dupuytren existe posiblemente desde hace siglos pero los historiadores médicos no han conseguido identificar alguna referencia de lesiones localizadas en la mano que se asemejaran a la enfermedad de Dupuytren. La descripción más antigua en la literatura médica parece ser la de Félix Plater (Figura 1). Nacido en Basel (Bâle), en Suiza, en el año 1536 (su nombre era Platter pero su padre Thomas Platterus latinizó su nombre suprimiendo una $t$, por lo que en algunas publicaciones aparece como Félix Platter); profesor de la Universidad de Basel; en el año 1571 fue nombrado Profesor Praxeos (profesor de medicina práctica) y Archiater (encargado de la dirección de los hospitales y jefe del servicio de salud pública de la ciudad de Basel), en el tercer volumen de su obra Observationum in hominis affectibus, publicada en Basel en el año
1614, año de su fallecimiento, se analizan 680 casos de enfermedades recogidos en 57 años de práctica médica, entre ellos se describe uno, caso 140 , que afectaba a un maestro picapedrero que presentaba una contracción del dedo anular y meñique de la mano izquierda (Figura 2).

El texto está escrito en un latín lleno de modismos que no sigue las reglas gramaticales correctas, por lo que se han hecho diversas interpretaciones (Elliot, 1988; Belusa et al, 1995; Belusa et al, 1997; Meinel, 1997), su traducción sería (Eugeni Ferré Cabré Prve. y Dr. Albert Bové Boada):

Contracción de los dedos de la mano izquierda hacia la palma.

Cierto famoso maestro picapedrero, cogiendo una gran piedra con la palma de la mano izquierda, se le bloquearon los tendones del 


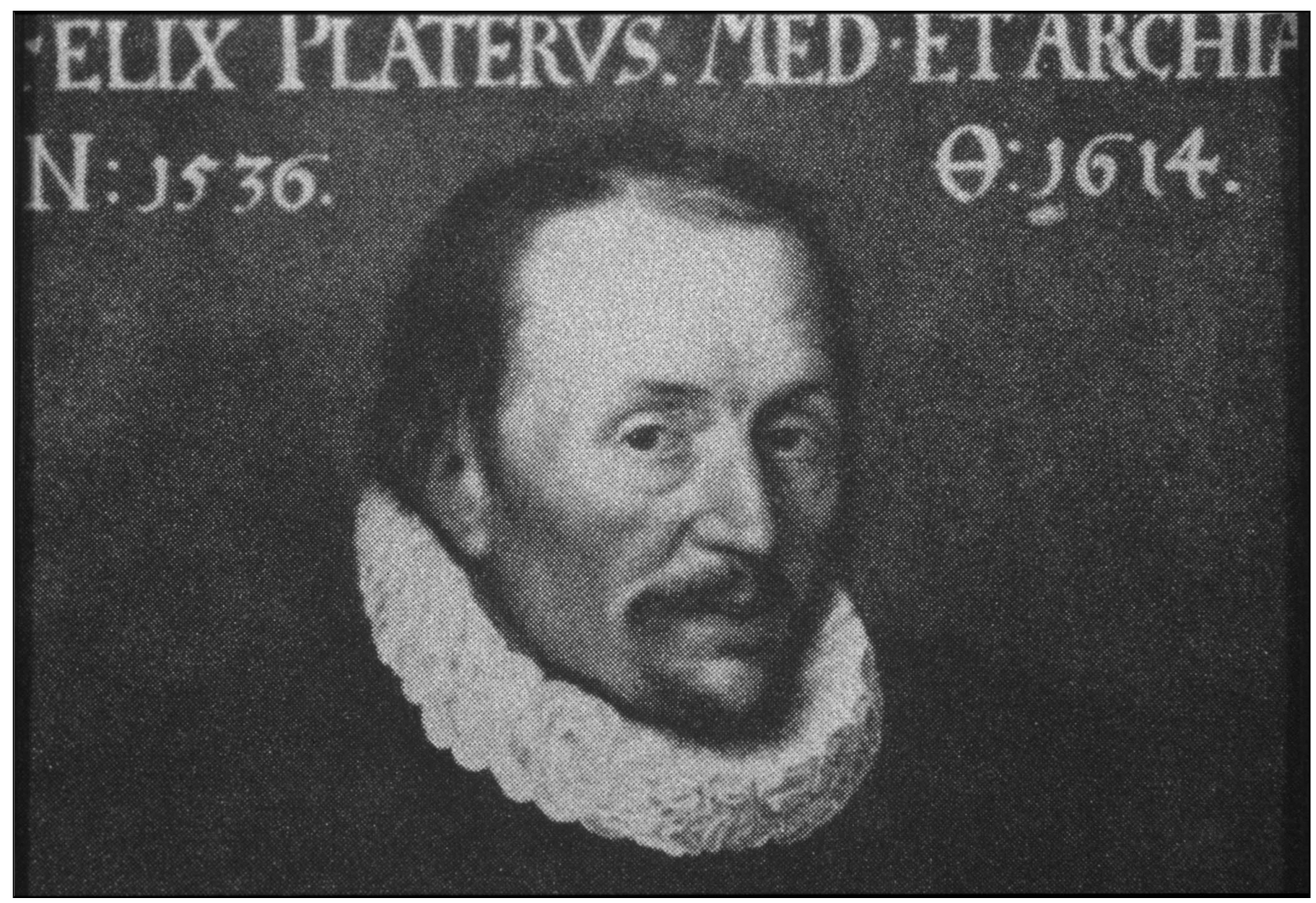

Figura 1. Félix Plater (1536-1614). Retrato realizado por von Hans Bock d. ̈̈. (1550-1624).

anular y meñique de tal manera que libres de sus vínculos quedaron elevados, formando dos cuerdas tensas bajo la piel, estos dos dedos permanecieron contraídos y traccionados para siempre.

Para Félix Plater la lesión era secundaria a un traumatismo, sin especificar la evolución desde el traumatismo hasta la contracción de los dedos. Describe el bloqueo de los tendones flexores, pero no queda claro si considera que

Contractio digitorum finiftra manus,
in volam illius.
Nfignis artifex lapicida quidam, faxum immen-
fum voluens, adeò téndines in finiftre manus
vola ad digitos, a nnulareın \& minimum definen.
tes, ei attracti funt, vt illi a vinculis quib.retinétur
laxati, eleuatiqúue, duas chordas fub cute tenfas in
altum referrent, contractiq́ue duo hi digiti \& at-
traeti, poftea femper manferint.

Figura 2. Reproducción de la descripción de Félix Plater, 1614. las cuerdas tensas bajo la piel son los tendones flexores u otras estructuras cordonales formadas en la palmar de la mano. Félix Plater conocía la aponeurosis palma puesto que en 1583 había publicado un libro titulado De Corporis Humani Sructura et Usu libri III, ilustrado con 50 tablas anatómicas inspiradas en Andreas Vesalius (De Humani Corporis Fabrica Libri septem, 1543) y en Volcher Coiter (Diversorum Animalium Sceletorum Explicationes, 1575), en las que se describe la aponeurosis palmar y sus extensiones subcutáneas, pero curiosamente en sus dibujos la aponeurosis palmar no llega al quinto dedo.

\section{AGRADECIMIENTOS}

A D. Joseph Ficher por la ayuda en la consecución y traducción de la biliografía, especialmente de los libros de Félix Plater. A D. Eugeni Ferré Cabré Prve. por la traducción e interpretación del texto en latín de la descripción de Félix Plater. 


\section{BIBLIOGRAFÍA}

Belusa, L.; Selzer, A. M.; Partechke, B. D.: Die Beschreibung der Dupuytren-Erkrankung durch den Baseler Arzt und Anatomen Felix Plater im Jahre 1614. Handchir Mikrochi Plast Chir, 1995; 27: 272275.

Belusa, L.; Kommentar zur Arbeit Meinel, A.; Belusa, L.; Selzer, A. M.; Partecke, B. D.: Die Beschreibung der DupuytrenErkrankung durch den Baseler Arzt und Anatomen Felix Plater im Jahre 1614. Handchir Mikrochir Plast Chir, 1995; 27: 272-275. Handchir Mikro- chir Plast Chir, 29: 1997; 109110.

Elliot, D.: The early history of contracture of the palmar fascia. Part 1: The origin of the disease: the curse of the MacCrimmons: the hand of benediction: Cline's contracture. J. Hand Surg, 13 (B): 246-253. 1988.

Meinel, A.: Kommentar zur Arbeit von Belusa, L.; Selzer, A. M.; Partecke, B. D.: Die Beschreibung der Dupuytren-Erkrankung durch den Baseler Arzt und Anatomen Felix Plater im Jahre 1614. Handchir Mikrochir Plast Chir, 1995; 27: 272-
275. Handchir Mikrochir Plast Chir, 1997; 29: 107-108.

Plater, F.: De Corporis Humani Structura et Usu. Libri III, Tabulis methodicè explicati, Iconibus accura tè illustrati. Ex officina Frobeniana. Per Ambrosium Frobenm Basileae, 1583; Cap. III, Fig. 14, 16 y 17.

Plater, F.: Observationum, In Hominis Affectibus plerisque, corpori et animo, functionum laesione, dolore aliáve molestiá et vitio incommodantibus, Libri Tres, Impensis Ludovici König, Basel, 1614; 140. 